\title{
A Unified Gas-kinetic Scheme for Continuum and Rarefied Flows
}

\author{
Kun $\mathrm{Xu}^{\mathrm{a}, *}$, Juan-Chen Huang ${ }^{\mathrm{b}}$ \\ ${ }^{a}$ Mathematics Department \\ Hong Kong University of Science and Technology \\ Clear Water Bay, Kowloon, Hong Kong \\ ${ }^{b}$ Department of Merchant Marine \\ National Taiwan Ocean University \\ Keelung 20224, Taiwan
}

\begin{abstract}
With discretized particle velocity space, a unified gas-kinetic scheme for entire Knudsen number flows is constructed based on the BGK model. In comparison with many existing kinetic schemes for the Boltzmann equation, the current method has no difficulty to get accurate solution in the continuum flow regime, such as the solution of the Navier-Stokes (NS) equations with the time step being much larger than the particle collision time, and the rarefied flow solution, even for the free molecule flow. The unified scheme is an extension of the gas-kinetic BGK-NS scheme from the continuum flow to the rarefied regime with the discretization of particle velocity space. The success of the method is due to the un-splitting treatment for the particle transport and collision in the evaluation of local solution of the gas distribution function. For these methods which use operator splitting technique to solve the transport and collision separately, it is usually required that the time step is less than the particle collision time, which basically makes these methods useless in the continuum flow regime, especially in the high Reynolds cases. Theoretically, once the physical process of particle transport and collision is modeled statistically by the gas-kinetic Boltzmann equation, the transport and collision become continuous operators in space and time, and their numerical discretization should be done consistently. With the use of the integral solution of the BGK, the unified scheme can simulate the flow accurately in the whole flow regime from the continuum Navier-Stokes solutions to the free molecule flow. At the same time, the time step in the high Reynolds number continuum flow region is only determined by the CFL condition of the macroscopic equations, instead of particle collision time.
\end{abstract}

Keywords: Unified scheme, Navier-Stokes equations, free molecule flow

\footnotetext{
${ }^{*}$ Corresponding author

Email addresses: makxu@ust.hk (Kun Xu), jchuang@mail.ntou.edu.tw (Juan-Chen Huang)
} 


\section{Introduction}

The development of accurate numerical methods for all flow regimes is challenging. An excellent example is the nozzle flow for controlling aerospace orbit, where both continuum and vacuum flows exist from gas container to the nozzle exit. Both the kinetic methods, such as direct simulation Monte Carlo (DSMC), and modern CFD techniques based on the Navier-Stokes (NS) equations, encounter computational difficulties when applied to these flows. The DSMC method requires that the time step and cell size are less than the particle collision time and mean free path, which subsequently introduce enormous computational cost in the high density regime. On the other hand, the conventional continuum NS methods are inapplicable for capturing non-equilibrium effects in the rarefied flow regime. High-order hydrodynamic equations are mostly limited to the transition flow regime only [19]. In recent years, the hybrid methods which combine NS and kinetic approaches have been often used to model flows which have both continuum and rarefied regimes [23, 9, 5, 26, 8]. A buffer zone is used to couple different approaches with the assumption of correctness of both methods in this zone. These approaches may sensitively depend on the location of the interface between different methods. Certainly, it is required that both methods are reliable in the buffer zone. But, in reality it may become that neither method can be applied there. For example, in many hybrid methods, it is assumed that in the buffer zone the flow can be correctly described by the NS equations, which means that the extension of the kinetic approach to the buffer zone should have the correct NS limit as well. But, this is just the difficult part for the kinetic approach. So, in order to accurately simulate the whole flow regimes, it is still desirable to have a single kinetic method which presents accurately the NS solution in the continuum flows and the collision-less Boltzmann solution in the free molecule regime. This can be done dynamically through the numerical discretization of the Boltzmann equation, instead of geometrically through the zone separations.

The Boltzmann equation describes the time evolution of the density distribution of a monatomic dilute gas with binary elastic collisions. The fluid dynamic Navier-Stokes (NS), Burnett and Super-Burnett equations can be derived from the Boltzmann equation. The Boltzmann equation is valid from the continuum flow regime to the free molecule flow. So, theoretically a unified kinetic method which is valid in the whole range of Knudsen number can be developed if the numerical discretization is properly designed. In the framework of deterministic approximation, the most popular class of methods is based on the so-called discrete velocity methods (DVM) or Discrete Ordinate Method (DOM) of the Boltzmann equation $[7,33,16,13,1,17]$. These methods use regular discretization of particle velocity space. Most of these methods can give accurate numerical solution for high Knudsen number 
flows, such as those from the upper transition to the free molecule regime. However, in the continuum flow regime, it is recognized that they have difficulty in the capturing of the Navier-Stokes solutions, especially for the high Reynolds number flows, where the intensive particle collisions take place. Under this situation, because the time step in these methods must be less than the particle collision time, which makes them prohibitive in the continuum flow application. In order to get unconditionally stable schemes with large time step, it is natural to use implicit or semi-implicit method for the collision part [13, 21, 11]. However, even though a scheme could overcome the stability restriction and use large time step, there is still accuracy concern, because many of the schemes have the same numerical mechanism as Flux Vector Splitting (FVS) methods in the continuum regime, where the intrinsic numerical dissipation is proportional to time step [30], which poisons the NS solution.

In order to develop a kinetic scheme for the whole flow regime, much effort has been paid on the development of the so-called asymptotic preserving (AP) scheme. As defined in [11], a kinetic scheme is AP if (1). it preserves the discrete analogy of the Chapman-Enskog expansion when the Knudsen numbers go to Zero. (2). in the continuum regime, the time step is not restricted by the particle collision time. Besides the above two conditions, we may need the third one as well, (3). the scheme has at least second-order accuracy in both continuum and free molecule regimes. Therefore, the AP scheme is the appropriate choice to solve the kinetic equation. For example, for the AP scheme in the nozzle simulation, with a uniform time step in the whole domain, this time step must be much larger than the particle collision time in the inner high density region, and be the same order or less in the outer rarefied and free molecular regions. Many approaches have been developed to develop kinetic AP methods $[2,9,11,21]$. To the current stage, it seems that the only AP scheme for the NS asymptotic in the continuum regime is the method developed by Bennoune, Lemou, and Mieussens [2]. Their deterministic method is based on a decomposition of the Boltzmann equation into a system which couples a kinetic equation (non-equilibrium) with a fluid one (equilibrium). The fluid part of this system degenerates, for small particle collision time, into the NS equations. However, in the collision-less limit, the physical basis of separating a distribution function into an equilibrium and a non-equilibrium part is questionable. The accuracy of the above scheme in the collision-less limit has to be tested as well. In other words, AP scheme should not only have the accuracy in the continuum flow regime, the validity of the kinetic method in the collision-less limit should be kept as well.

In order to develop an AP scheme for the kinetic equation, we need a correct understanding of the Boltzmann equation. Even though the individual particle movement has distinct transport and collision process, once this process is described by the statistical model, such as the Boltzmann equation, the transport and collision processes are coupled everywhere in space and time. To separate them numerically, such as the operator splitting methods, is inconsistent with the underlying physical model. As shown in this paper, a simple up- 
winding discretization for the particle transport term in the Botltzmann equation, i.e., $u f_{x}$ term, is basically an operator splitting scheme and introduces difficulties to get AP property. Without correctly discretizing this term, any other modification in the kinetic method may not work properly in the whole flow regime. In the high Knudsen number flow regime, the decoupling of transport and collision may not become a problem, because the numerical error introduced due to the decoupling (being proportional to time step) is much less than the physical one (being proportional to particle collision time). But, it cannot be tolerated in the continuum flow regime, especially for an AP method.

In the past years, the gas-kinetic BGK-NS scheme for the Navier-Stokes solutions has been well developed [29], and has been successfully applied for the continuum flow simulations from nearly incompressible to hypersonic viscous and heat conducting flows $[25,31,32,12,15]$. In the BGK-NS method, the particle velocity space is continuous and is integrated out in the flux evaluations in a finite volume scheme. This is not surprised because in the fluid regime, based on the Chapman-Enskog expansion the gas distribution function for the viscous flow is well-defined. Therefore, the efficiency of the BGK-NS method is similar to the traditional NS flow solver, where the same CFL condition is used for the determination of time step. Theoretically, any kinetic scheme with NS asymptotic AP property should be able to recover the BGK-NS scheme in the continuum limit. So, different from other approaches, in this paper we are going to extend the BGK-NS method for the continuum flow to the rarefied regime, which includes the free molecule limit. In order to do that, we have to discretize the particle velocity space as well, because the real gas distribution function in the highly non-equilibrium region can be hardly described by a Maxwellian distribution function and its derivatives. So, the current method can be also considered as a discrete velocity version of the BGK-NS scheme.

\section{Kinetic theory and Discrete Ordinate Method}

In this paper, we will present the unified scheme for all Knudsen number flows. The one-dimensional kinetic equation will be used to illustrate the idea. In this section, we are going to first introduce the kinetic equation and the traditional discrete ordinate method (DOM). In the next section, the unified method will be presented.

The one-dimensional gas-kinetic BGK equation can be written as $[6,3]$

$$
f_{t}+u f_{x}=\frac{g-f}{\tau}
$$

where $f$ is the gas distribution function and $g$ is the equilibrium state approached by $f$. Both $f$ and $g$ are functions of space $x$, time $t$, particle velocities $u$, and internal variable $\xi$. 
The particle collision time $\tau$ is related to the viscosity and heat conduction coefficients. The equilibrium state is a Maxwellian distribution,

$$
g=\rho\left(\frac{\lambda}{\pi}\right)^{\frac{K+1}{2}} e^{-\lambda\left((u-U)^{2}+\xi^{2}\right)},
$$

where $\rho$ is the density, $U$ is the macroscopic velocity in the $x$ direction, $\lambda$ is equal to $m / 2 k T$, $m$ is the molecular mass, $k$ is the Boltzmann constant, and $T$ is the temperature. For $1 D$ flow, the total number of degrees of freedom $K$ in $\xi$ is equal to $(3-\gamma) /(\gamma-1)$. For example, for a diatomic gas with $\gamma=7 / 5, K$ is equal to 4 to account for the particle motion in the $y$ and $z$-directions, as well as two rotational degrees of freedom. In the equilibrium state, the internal variable $\xi^{2}$ is equal to $\xi^{2}=\xi_{1}^{2}+\xi_{2}^{2}+\ldots+\xi_{K}^{2}$. The relation between mass $\rho$, momentum $\rho U$, and energy $\rho E$ densities with the distribution function $f$ is

$$
\left(\begin{array}{c}
\rho \\
\rho U \\
\rho E
\end{array}\right)=\int \psi_{\alpha} f d \Xi, \quad \alpha=1,2,3,
$$

where $\psi_{\alpha}$ is the component of the vector of moments

$$
\boldsymbol{\psi}=\left(\psi_{1}, \psi_{2}, \psi_{3}\right)^{T}=\left(1, u, \frac{1}{2}\left(u^{2}+\xi^{2}\right)\right)^{T}
$$

and $d \Xi=d u d \xi_{1} d \xi_{2} \ldots d \xi_{K}$ is the volume element in the phase space with $d \xi=d \xi_{1} d \xi_{2} \ldots d \xi_{K}$. Since mass, momentum, and energy are conserved during particle collisions, $f$ and $g$ satisfy the conservation constraint,

$$
\int(g-f) \psi_{\alpha} d \Xi=0, \quad \alpha=1,2,3,
$$

at any point in space and time.

Before we introduce discrete ordinate method, let's first discretize the space, time, and particle velocity. For a numerical cell $j$ in space, i.e., $x \in\left[x_{j-1 / 2}, x_{j+1 / 2}\right]$, at the time level $t^{n}$, and at discretized particle velocity interval $k$, i.e., $u \in\left[u_{k}-\frac{1}{2} \Delta u, u_{k}+\frac{1}{2} \Delta u\right]$, the phase space averaged partilcle distribution function becomes

$$
f\left(x_{j}, t^{n}, u_{k}\right)=f_{j, k}^{n}=\frac{1}{\Delta x \Delta u} \int_{x_{j-1 / 2}}^{x_{j+1 / 2}} \int_{u_{k}-\frac{1}{2} \Delta u}^{u_{k}+\frac{1}{2} \Delta u} f\left(x, t^{n}, u\right) d x d u,
$$

where $\Delta u$ is the particle velocity interval and $\Delta x=x_{j+1 / 2}-x_{j-1 / 2}$ is the cell size. 
The BGK equation (1) can be written as

$$
f_{t}=-u f_{x}+\frac{g-f}{\tau} .
$$

Integrating the above equation in a control volume $\int_{x_{j-1 / 2}}^{x_{j+1 / 2}} \int_{t^{n}}^{t^{n+1}} d x d t$, and keeping the particle velocity space continuous, the differential equation becomes an integral equation

$$
f_{j}^{n+1}=f_{j}^{n}+\frac{1}{\Delta x} \int_{t^{n}}^{t^{n+1}}\left(u f_{x_{j-1 / 2}}(t)-u f_{x_{j+1 / 2}}(t)\right) d t+\frac{1}{\Delta x} \int_{t^{n}}^{t^{n+1}} \int_{x_{j-1 / 2}}^{x_{j+1 / 2}} \frac{g-f}{\tau} d x d t,
$$

where $f_{j+1 / 2}$ is the gas distribution function at the cell interface $x_{j+1 / 2}$. The above equation is exact and there is no any numerical error introduced yet. For a kinetic scheme, two terms on the right hand side of the above equation have to be numerically evaluated.

A standard operator splitting method is to solve the above equation in the following steps $[8,33]$. The equation $(6)$ is decoupled to

$$
f_{j}^{*}=f_{j}^{n}+\frac{1}{\Delta x} \int\left(u f_{x_{j-1 / 2}}(t)-u f_{x_{j+1 / 2}}(t)\right) d t,
$$

for the transport across a cell interface, and

$$
f_{t}=\frac{g-f}{\tau},
$$

for the particle collision or source term inside each cell. In the transport part, the collisionless Boltzmann equation or upwinding approach,

$$
f_{t}+u f_{x}=0,
$$

is used for the flux evaluation at the cell interface. For example, for a 1st-order method, the upwinding flux is based on the following solution at the cell interface,

$$
f_{j+1 / 2}= \begin{cases}f_{j}, & u \geq 0 \\ f_{j+1}, & u<0\end{cases}
$$

which is dynamically equivalent to the flux vector splitting scheme. The above transport and collision steps can be also coupled using Runge-Kutta method within a time step.

If we take conservative moments $\psi_{\alpha}$ on Eq.(6), due to the conservation of conservative variables during particle collision step, the update of conservative variables becomes

$$
W_{j}^{n+1}=W_{j}^{n}+\frac{1}{\Delta x} \int_{t^{n}}^{t^{n+1}}\left(F_{x_{j-1 / 2}}(t)-F_{x_{j+1 / 2}}(t)\right) d t,
$$


where $W$ is the averaged conservative mass, momentum, and energy densities inside each cell, and $F=\int u \psi_{\alpha} f_{0}(x-u t) d \Xi$ is the flux based on the collisionless Boltzmann equation. The above scheme for the update of macroscopic flow variables is physically identical to the kinetic flux vector splitting schemes (KFVS) $[22,10]$. The only difference is the initial gas distribution function $f_{0}$ at the beginning of each time step. For the KFVS scheme, an equilibrium state is prepared for $f_{0}$, and for the DOM $f_{0}$ is explicitly updated from the equation with discretized particle velocity space. But, this will not change the numerical dissipative mechanism in the KFVS method, where the numerical dissipation is proportional to time step. Therefore, if a large time step could be used for the DOM method in the continuum flow regime, the numerical dissipation would also make DOM scheme be inaccurate for the NS solutions. It is a well-known defect for all Flux Vector Splitting methods [27]. So, in the continuum flow regime, for the DOM method the requirement of time step being less than the particle collision time is not only for the stability consideration, but also a necessary condition for the accuracy. But, this constraint deviates the above scheme away from an AP method for the NS solutions.

In order to design an AP scheme which is valid for both NS equations and the free molecule limit, the full BGK equation has to be solved in the flux evaluation in Eq.(6) across a cell interface,

$$
f_{t}+u f_{x}=\frac{g-f}{\tau}
$$

The necessity of using the above equation can be understood in the following. First, let's forget about numerical mesh. Since the Boltzmann equation couples the particle transport and collision everywhere, at any point in space and time $(x, t)$, both collision and transport exist. For example, $u f_{x}(x, t)$ and $(g-f) / \tau$ are continuous function of $x$ and $t$. Now let's put the mesh into the space. For these molecules, if they move in a zig-zag way from one place to another place in a time step within the same cell, in terms of the cell resolution these molecules will only contribute to the modification of particle distribution function through the particle collisions, such as $f_{t}=(g-f) / \tau$. However, for those zig-zag movement molecules, within a time step if they pass through the cell interface, their contributions will be the first term on the right hand side of Eq.(6). Physically, the dynamics for those molecules within a cell or across a cell interface should be the same, and both transport and collision exist in their movements. In this way, those molecules passing through cell interface do suffer from particle collision during their passage moving towards the cell interface.

\section{Unified Kinetic Scheme in all Flow Regimes}

In this section, we will present a unified kinetic scheme based on the BGK model for all flow regimes. The current method is a natural extension of the gas-kinetic BGK-NS method 
from the compressible Navier-Stokes equations to all Knudsen number flow. In this section, only one-dimensional formulation will be presented. The extension to 2D and 3D cases are straightforward for the kinetic method.

With the discretization of space $x_{j}$, time $t^{n}$, and particle velocity $u_{k}$, the finite volume solution of the kinetic equation (6) is

$$
f_{j, k}^{n+1}=f_{j, k}^{n}+\frac{1}{\Delta x} \int\left(u_{k} f_{j-1 / 2, k}-u_{k} f_{j+1 / 2, k}\right) d t+\frac{1}{\Delta x} \iint \frac{g-f}{\tau} d x d t .
$$

where $f_{j, k}^{n}$ is the cell averaged distribution function in $j t h$-cell $x \in\left[x_{j-1 / 2}, x_{j+1 / 2}\right]$ at particle velocity $u_{k}$. Instead of using upwinding scheme for the evaluation of the distribution function at a cell interface, the solution $f_{j+1 / 2, k}$ in the above equation is constructed from the integral solution of the BGK model,

$$
\begin{aligned}
f_{j+1 / 2, k}= & f\left(x_{j+1 / 2}, t, u_{k}, \xi\right)=\frac{1}{\tau} \int_{0}^{t} g\left(x^{\prime}, t^{\prime}, u_{k}, \xi\right) e^{-\left(t-t^{\prime}\right) / \tau} d t^{\prime} \\
& +e^{-t / \tau} f_{0, k}\left(x_{j+1 / 2}-u_{k} t\right)
\end{aligned}
$$

where $x^{\prime}=x_{j+1 / 2}-u\left(t-t^{\prime}\right)$ is the particle trajectory. In the above equation, the distribution function $f_{0}$ at particle velocity $u_{k}$ should be given initially at the beginning of each time step, and a high-order reconstruction can be used to construct its subcell resolution, such as using TVD and ENO methods. In order to simplify the notation, in the following the cell interface $x_{j+1 / 2}=0$ and $t^{n}=0$ are used. For example, around each cell interface $x_{j+1 / 2}$, at time step $t^{n}$ the initial distribution function becomes,

$$
f_{0}\left(x, t^{n}, u_{k}, \xi\right)=f_{0, k}(x, 0)= \begin{cases}f_{j+1 / 2, k}^{l}\left[1+\sigma_{j, k} x\right], & x \leq 0 \\ f_{j+1 / 2, k}^{r}\left[1+\sigma_{j+1, k} x\right], & x>0\end{cases}
$$

where nonlinear limiter can be used to reconstruct $f_{j+1 / 2, k}^{l}, f_{j+1 / 2, k}^{r}$ and the corresponding slopes $\sigma_{j, k}$. For the integral solution of the equilibrium state, since the macroscopic variables inside each cell are known, we can first use a continuous particle velocity space to evaluate the integral. For an equilibrium state $g$ around a cell interface $\left(x_{j+1 / 2}=0, t=0\right)$, same as the BGK-NS scheme, it can be expanded with two slopes [29],

$$
g=g_{0}\left[1+(1-\mathrm{H}[x]) \bar{a}^{l} x+\mathrm{H}[x] \bar{a}^{r} x+\bar{A} t\right],
$$

where $\mathrm{H}[x]$ is the Heaviside function defined as

$$
H[x]= \begin{cases}0, & x<0 \\ 1 . & x \geq 0\end{cases}
$$


Here $g_{0}$ is a local Maxwellian distribution function located at $x=0$. Even though, $g$ is continuous at $x=0$, but it has different slopes at $x<0$ and $x>0$. In $g, \bar{a}^{l}, \bar{a}^{r}$, and $\bar{A}$ are related to the derivatives of a Maxwellian distribution in space and time. In the calculation of the equilibrium state in space and time, it is not necessary to use a distribution function with discretized velocity space. Based on the macroscopic flow distribution functions inside each cell, we can construct its solution with a continuous particle velocity space first, then take its corresponding value at the specific particle velocity when necessary. The expansion of the above equilibrium distribution is coming from a Taylor expansion of a Maxwellian in space and time. In order to have AP scheme for the Navier-Stokes equations, to keep the 1st-order expansion of an equilibrium state in space and time is necessary. Certainly, if you would like to have a higher than second-order accuracy scheme, a high-order expansion needs to be used [15].

The dependence of $\bar{a}^{l}, \bar{a}^{r}$ and $\bar{A}$ on the particle velocities can be obtained from a Taylor expansion of a Maxwellian and have the following form,

$$
\begin{aligned}
& \bar{a}^{l}=\bar{a}_{1}^{l}+\bar{a}_{2}^{l} u+\bar{a}_{3}^{l} \frac{1}{2}\left(u^{2}+\xi^{2}\right)=\bar{a}_{\alpha}^{l} \psi_{\alpha}, \\
& \bar{a}^{r}=\bar{a}_{1}^{r}+\bar{a}_{2}^{r} u+\bar{a}_{3}^{r} \frac{1}{2}\left(u^{2}+\xi^{2}\right)=\bar{a}_{\alpha}^{r} \psi_{\alpha}, \\
& \bar{A}=\bar{A}_{1}+\bar{A}_{2} u+\bar{A}_{3} \frac{1}{2}\left(u^{2}+\xi^{2}\right)=\bar{A}_{\alpha} \psi_{\alpha},
\end{aligned}
$$

where $\alpha=1,2,3$ and all coefficients $\bar{a}_{1}^{l}, \bar{a}_{2}^{l}, \ldots, \bar{A}_{3}$ are local constants.

The determination of $g_{0}$ depends on the determination of the local macroscopic values of $\rho_{0}, U_{0}$ and $\lambda_{0}$ in $g_{0}$, i.e.,

$$
g_{0}=\rho_{0}\left(\frac{\lambda_{0}}{\pi}\right)^{\frac{K+1}{2}} e^{-\lambda_{0}\left(\left(u-U_{0}\right)^{2}+\xi^{2}\right)}
$$

which can be determined uniquely using the compatibility condition of the BGK model. Taking the limit $t \rightarrow 0$ in Eq.(14) and substituting its solution into Eq.(3), the conservation constraint at $\left(x=x_{j+1 / 2}, t=0\right)$ gives

$$
W_{0}=\int g_{0} \boldsymbol{\psi} d \Xi=\sum_{k}\left(f_{j+1 / 2, k}^{l} H\left(u_{k}\right)+f_{j+1 / 2, k}^{r}\left(1-H\left(u_{k}\right)\right)\right) \boldsymbol{\psi}
$$

where $W_{0}=\left(\rho_{0}, \rho_{0} U_{0}, E_{0}\right)^{T}$ is the macroscopic conservative flow variables located at the cell interface at time $t=0$. Since $f_{j+1 / 2, k}^{l}$ and $f_{j+1 / 2, k}^{r}$ have been obtained earlier in the initial distribution function $f_{0}$ around a cell interface, the above moments can be evaluated explicitly. Therefore, the conservative variables $\rho_{0}, \rho_{0} U_{0}$, and $E_{0}$ at the cell interface can be 
obtained, from which $g_{0}$ is uniquely determined. Different from the BGK-NS method, the integration of $f_{0}$ term in BGK-NS is replaced by the sum in particle velocity due to the discretization of particle velocity space. Also, due to the particle velocity discretization, the gas distribution function has more freedom to recover any distribution function in the highly non-equilibrium flow regime and the governing equations underlying the current approach can be beyond the NS in the general case. For the equilibrium state, $\lambda_{0}$ in $g_{0}$ can be found from

$$
\lambda_{0}=(K+1) \rho_{0} /\left(4\left(E_{0}-\frac{1}{2} \rho_{0} U_{0}^{2}\right)\right) .
$$

Then, $\bar{a}^{l}$ and $\bar{a}^{r}$ of $g$ in Eq.(16) can be obtained through the relation of

$$
\frac{\bar{W}_{j+1}\left(x_{j+1}\right)-W_{0}}{\rho_{0} \Delta x^{+}}=\int \bar{a}^{r} g_{0} \psi d \Xi=\bar{M}_{\alpha \beta}^{0}\left(\begin{array}{c}
\bar{a}_{1}^{r} \\
\bar{a}_{2}^{r} \\
\bar{a}_{3}^{r}
\end{array}\right)=\bar{M}_{\alpha \beta}^{0} \bar{a}_{\beta}^{r},
$$

and

$$
\frac{W_{0}-\bar{W}_{j}\left(x_{j}\right)}{\rho_{0} \Delta x^{-}}=\int \bar{a}^{l} g_{0} \psi d \Xi=\bar{M}_{\alpha \beta}^{0}\left(\begin{array}{c}
\bar{a}_{1}^{l} \\
\bar{a}_{2}^{l} \\
\bar{a}_{3}^{l}
\end{array}\right)=\bar{M}_{\alpha \beta}^{0} \bar{a}_{\beta}^{l},
$$

where the matrix $\bar{M}_{\alpha \beta}^{0}=\int g_{0} \psi_{\alpha} \psi_{\beta} d \Xi / \rho_{0}$ is known, and $\Delta x^{+}=x_{j+1}-x_{j+1 / 2}$ and $\Delta x^{-}=$ $x_{j+1 / 2}-x_{j}$ are the distances from the cell interface to cell centers. Therefore, $\left(\bar{a}_{1}^{r}, \bar{a}_{2}^{r}, \bar{a}_{3}^{r}\right)^{T}$ and $\left(\bar{a}_{1}^{l}, \bar{a}_{2}^{l}, \bar{a}_{4}^{l}\right)$ can be found following the procedure as BGK-NS method [29]. In order to evaluate the time evolution part $\bar{A}$ in the equilibrium state, we can apply the following condition

$$
\frac{d}{d t} \int(g-f) \psi \Xi=0
$$

at $(x=0, t=0)[14]$ and obtained

$$
\begin{aligned}
\bar{M}_{\alpha \beta}^{0} \bar{A}_{\beta} & =(\partial \rho / \partial t, \partial(\rho U) / \partial t, \partial E / \partial t)^{T} \\
& =\frac{1}{\rho_{0}} \int\left[u\left(\bar{a}^{l} \mathrm{H}[u]+\bar{a}^{r}(1-\mathrm{H}[u])\right) g_{0}\right] \boldsymbol{\psi} d \Xi .
\end{aligned}
$$

Up to this point, we have determined all parameters in the initial gas distribution function $f_{0}$ and the equilibrium state $g$ at the beginning of each time step $t=0$. After substituting Eq.(15) and Eq.(16) into Eq.(14), the gas distribution function $f\left(x_{j+1 / 2}, u_{k}, t\right)$ at the discretized particle velocity $u_{k}$ can be expressed as

$$
\begin{aligned}
f_{j+1 / 2, k}(0, t)= & \left(1-e^{-t / \tau}\right) g_{0} \\
& +\left(\tau\left(-1+e^{-t / \tau}\right)+t e^{-t / \tau}\right)\left(\bar{a}^{l} \mathrm{H}\left[u_{k}\right]+\bar{a}^{r}\left(1-\mathrm{H}\left[u_{k}\right]\right)\right) u_{k} g_{0}
\end{aligned}
$$




$$
\begin{aligned}
& +\tau\left(t / \tau-1+e^{-t / \tau}\right) \bar{A} g_{0} \\
& +e^{-t / \tau}\left(\left(1-u_{k} t \sigma_{j, k}\right) \mathrm{H}\left[u_{k}\right] f_{j+1 / 2, k}^{l}+\left(1-u_{k} t \sigma_{j+1, k}\right)\left(1-\mathrm{H}\left[u_{k}\right]\right) f_{j+1 / 2, k}^{r}\right) \\
= & \tilde{g}_{j+1 / 2, k}+\tilde{f}_{j+1 / 2, k},
\end{aligned}
$$

where $\tilde{g}_{j+1 / 2, k}$ is all terms related to the integration of the equilibrium state $g$ and $\tilde{f}_{j+1 / 2, k}$ is the terms from initial condition $f_{0}$ in the integral solution. The above time-accurate gas distribution function can be used in Eq.(13) for the fluxes at a cell interface. In order to solve Eq.(13) for the gas distribution function $f_{j, k}^{n+1}$, we can first take moment $\boldsymbol{\psi}$ on Eq.(13). Due to the vanishing of the particle collision term for the conservative variables, we have

$$
\begin{aligned}
W_{j}^{n+1}= & W_{j}^{n}+\frac{1}{\Delta x} \iint_{t^{n}}^{t^{n+1}} u\left(\tilde{g}_{j-1 / 2, k}-\tilde{g}_{j+1 / 2, k}\right) \boldsymbol{\psi} d t d u \\
& +\frac{1}{\Delta x} \sum_{k} \int_{t^{n}}^{t^{n+1}} u_{k}\left(\tilde{f}_{j-1 / 2, k}-\tilde{f}_{j+1 / 2, k}\right) \boldsymbol{\psi} d t
\end{aligned}
$$

where the integration of the equilibrium part $\tilde{g}$ can be evaluated exactly in a continuous particle velocity space and the integration of the non-equilibrium part $\tilde{f}$ can be done using the quadrature. For the update of the conservative variables, the difference between the above formulation and the BGK-NS scheme is that the discrete sum is used for the integration of the initial distribution function $f_{0}$ in particle velocity space. For a highly non-equilibrium flow, the real distribution function $f_{0}$ can be a complicated function, and a discrete version with the discretization of particle velocity space has to be used. For the original BGK-NS scheme [29], since the NS solutions are the target, the initial gas distribution function $f_{0}$ can be reconstructed from the distribution of macroscopic variables according to the ChapmanEnskog expansion. Therefore, the specific form of initial condition $f_{0}$ can be mathematically described using simple function. In the continuum limit, due to the sufficient number of particle collisions and with the condition of time step being much larger than the particle collision time, the integration of the equilibrium part $\tilde{g}_{j-1 / 2}$ and $\tilde{g}_{j+1 / 2}$ will be dominant and gives a corresponding NS distribution function by itself [28], and the contribution from initial terms $\tilde{f}_{j-1 / 2, k}$ and $\tilde{f}_{j+1 / 2, k}$ vanishes. As a result, the updated discrete form of the distribution function $f_{j, k}^{n+1}$ will present a Chapman-Enskog NS distribution function. Therefore, in the continuum flow regime, the BGK-NS scheme with continuous particle velocity space and the current unified method with discretized particle velocity space will become the same scheme. In the continuum flow regime, for the NS solutions only the update of the conservative variables through the above equation (22) is enough, because the gas distribution function $f_{j, k}^{n+1}$ can be constructed from the updated conservative variables. Therefore, for the continuum only, like the BGK-NS scheme [29], we don't basically update the gas distribution function. 
This also illustrate that in terms of the conservative variables update, the unified scheme is an AP method in the continuum regime. However, the unified scheme is not limited to continuum flow. Even in the highly non-equilibrium flow regime, Equation (22) for the update of conservative variables is still correct. For example, in the collisionless limit, the non-equilibrium part $\tilde{f}_{j-1 / 2, k}$ and $\tilde{f}_{j+1 / 2, k}$ will take dominant effect, and the contribution from the equilibrium part vanishes. Therefore, the unified scheme has the collision-less limit correct as well.

In general, based on the above updated conservative variables, we can immediately obtain the equilibrium gas distribution function $g_{j, k}^{n+1}$, therefore the unified kinetic scheme for the update of gas distribution function inside each cell can be written as

$$
\begin{aligned}
f_{j, k}^{n+1}= & f_{j, k}^{n}+\frac{1}{\Delta x}\left(\int_{t^{n}}^{t^{n+1}} u_{k}\left(\tilde{g}_{j-1 / 2, k}-\tilde{g}_{j+1 / 2, k}\right) d t+\int_{t^{n}}^{t^{n+1}} u_{k}\left(\tilde{f}_{j-1 / 2, k}-\tilde{f}_{j+1 / 2, k}\right) d t\right) \\
& +\frac{\Delta t}{2}\left(\frac{g_{j, k}^{n+1}-f_{j, k}^{n+1}}{\tau^{n+1}}+\frac{g_{j, k}^{n}-f_{j, k}^{n}}{\tau^{n}}\right)
\end{aligned}
$$

where trapezoidal rule has been used for the time integration of collision term. Since the conservative variables at time step $t^{n+1}$ have been obtained, the corresponding local particle collision time $\tau^{n+1}$ can be evaluated as well. So, from the above equation, a unified scheme for the update of gas distribution function becomes

$$
\begin{aligned}
f_{j, k}^{n+1}= & \left(1+\frac{\Delta t}{2 \tau^{n+1}}\right)^{-1}\left[f_{j, k}^{n}+\frac{1}{\Delta x}\left(\int_{t^{n}}^{t^{n+1}} u_{k}\left(\tilde{g}_{j-1 / 2, k}-\tilde{g}_{j+1 / 2, k}\right) d t\right.\right. \\
& \left.\left.+\int_{t^{n}}^{t^{n+1}} u_{k}\left(\tilde{f}_{j-1 / 2, k}-\tilde{f}_{j+1 / 2, k}\right) d t\right)+\frac{\Delta t}{2}\left(\frac{g_{j, k}^{n+1}}{\tau_{j}^{n+1}}+\frac{g_{j, k}^{n}-f_{j, k}^{n}}{\tau_{j}^{n}}\right)\right],
\end{aligned}
$$

where no iteration is needed for the update of the above solution.

\section{Physical and Numerical Analysis}

In the previous section, we present a unified kinetic scheme based on the kinetic BGK model. The scheme is a natural extension of BGK-NS scheme for the NS solutions to the rarefied flow regimes with discretized particle velocity space. The scheme can be further understood in the following aspects.

The scheme presented in the last section can be considered as a hybrid scheme between macroscopic and microscopic approaches. The traditional hybrid approach is based on a geometrical approach. In different flow regions, different governing equations are solved. At 
the same time, different patches are connected through buffer zone. However, instead of solving different governing equations as most hybrid schemes do, we couple them in the way of evaluating the flux function across the cell interface. In the continuum flow regime, the intensive particle collision will drive the system close to equilibrium state. Therefore, the part based on the integration of equilibrium state $\tilde{g}_{j+1 / 2, k}$ in Eq.(21) at the cell interface will automatically take a dominant role. It can be shown that in smooth flow region $\tilde{g}_{j+1 / 2, k}$ gives precisely the NS gas distribution function. Since there is one-to-one correspondence between macroscopic flow variables and the equilibrium distribution, the integration of the equilibrium one can be also fairly considered as a macroscopic composition part of the scheme. In the free molecule limit with inadequate particle collisions, the integral solution at the cell interface will automatically present a purely upwinding scheme, where the particle transport from $\bar{f}_{j+1 / 2, k}$ will be the main part. Therefore, the scheme also captures the flow physics in the collisionless limit. This kind of hybrid approach can be considered as a dynamic one instead of geometrical one. The reason for most approaches to use a geometrical way is due to the fact that their fluxes function across a cell interface are solely based on the kinetic upwinding discretization, i.e., the so-called $\tilde{f}_{j+1 / 2, k}$ term in Eq.(21). As we know, the kinetic upwinding is only correct in the collisionless or highly non-equilibrium regime. In the traditional hybrid scheme, the computational domain has to be divided into equilibrium and non-equilibrium flow regions. Physically this kind of geometrical division is artificial and there should have no region where both approaches are applicable, because the above two approaches have significant dynamic differences in their flux evaluation. In our case, we have a single computation domain and the dynamic differences in the particle behavior is obtained by solving the full approximate Boltzmann equation, which is valid all the way from the continuum to rarefied flows.

Asymptotic Preserving for the NS solution in the continuum limit is a preferred property for the kinetic scheme. In order to design an AP scheme which is valid for both NS equations and the free molecule limit, it is necessary to solve the full BGK equation for the flux evaluation in Eq.(6) across a cell interface,

$$
f_{t}+u f_{x}=\frac{g-f}{\tau} .
$$

The necessity of using the above equation can be understood in the following. First, let's forget about numerical mesh. Since the Boltzmann equation couples the particle transport and collision everywhere, at any point in space and time $(x, t)$, both collision and transport exist. For example, $u f_{x}(x, t)$ and $(g-f) / \tau$ are continuous function of $x$ and $t$. When evaluating particle transport across a cell interface, dynamically all particles in a small domain around the cell interface will effect the particle evolution through transport and collision. In other words, those molecules passing through cell interface do suffer from particle 
collisions as well. The collisionless or upwinding discretization which accounts only for $\tilde{f}_{j+1 / 2, k}$ term in Eq. $(21)$ is physically incorrect.

As shown in the last section, the distinguishable point of the current scheme is the collisional BGK is solved for the flux evaluation at a cell interface. For the continuum flow regime, only conservative flow variables are concerned. The flux evaluation for the conservative variables update in Eq.(22) is simply a discretized version of the BGK-NS method for the NS equations. For the unified scheme, for the continuum flow, we can use the time step which is much larger than the particle collision time, i.e., $\Delta t>>\tau$. In this case, the distribution function for the flux evaluation at the cell interface Eq.(21) will become

$$
f_{j+1 / 2}(t)=g_{0}(1-\tau(\bar{a} u+\bar{A})+t \bar{A})
$$

which is exactly the Chapman-Enskog expansion of the NS solution [29], and where $\bar{a}=$ $\bar{a}^{l} H\left[u_{k}\right]+\bar{a}^{r}\left(1-H\left[u_{k}\right]\right)$. In other words, in the continuum limit, the integral solution from the equilibrium state is dominant, and the updating of the macroscopic flow variables follow the NS solutions. In other words, the unified scheme provides an accurate NS solutions in the update of the conservative variables inside each cell. It is an AP method and the accuracy of the unified scheme in the continuum flow regime is $O\left(\tau(\Delta t)^{2}\right)[18,20]$. Theoretically, in the continuum limit it is not necessary to evaluate the distribution function anymore. If a simple upwinding method is used for the flux evaluation, as done by most kinetic methods, an artificial viscosity term being proportional to time step $\Delta t$ will be introduced in its governing equations. So, in this case, the kinetic scheme can only become an asymptotic preserving method for the Euler equations only.

In the free molecule limit, i.e., $\tau \rightarrow \infty$, the unified scheme will become

$$
f_{j, k}^{n+1}=f_{j, k}^{n}-\int_{t^{n}}^{t^{n+1}} u_{k}\left(\bar{f}_{j-1 / 2, k}-\bar{f}_{j+1 / 2, k}\right) d t,
$$

where only the initial term $f_{0}$ in the integral solution of the BGK model Eq.(14) appears. The above equation is a purely free molecule transport equation. So, in this limit the unified kinetic scheme is a valid one.

\section{Numerical Experiments}

The development of accurate kinetic methods for simulating flows in entire Knudsen number regime is difficult. The discrete ordinate methods (DOM) of Yang-Huang, Mieussens, and Li-Zhang [33, 13, 16] are accurate numerical methods in all Knudsen numbers, but the limitation of the time step on these schemes in the continuum flow regime makes them impossible be applied in computations. But, still the results from DOM methods can be 
used as a benchmark solution for these flows if the computational cost is affordable. This is also true all many other kinetic methods, such as DSMC and direction Boltzmann solver $[4,1]$.

In this section, in order to test the unified scheme we are going to present two onedimensional test cases for free molecule flow to continuum Euler and NS solutions. The flow features of different local Knudsen numbers will be covered. In the following, we are going to test three schemes, which are the unified scheme (unified BGK), discrete ordinate method (BGK-DOM) [33], and the continuum flow solver BGK-NS for NS solutions [29]. The unified scheme will be applied to all Knudsen number flow regime. BGK-DOM will be applied to rarefied and near continuum flows where it is applicable. The BGK-NS will be applied to near continuum and continuum flows. For the unified BGK method, in all test cases the time step is determined by the CFL condition with CFL number 0.9. For the BGK-DOM, the time step is limited by the particle collision time, which is on the order of $\Delta t \leq 2 \tau_{m}$, where $\tau_{m}$ is the minimum particle collision time in the domain. For the BGK-NS code, the time step is also determined by the CFL condition.

The first case is the standard Sod test. In the computational domain $x \in[0,1], 100$ cells with uniform mesh in space are used. In the particle velocity space, 200 points with uniform distribution are used. Certainly, this number can be much reduced. But, the propose here is to test the code instead of optimizing the velocity space. The initial condition for the mass density, velocity, and pressure is

$$
\begin{aligned}
\left(\rho_{l}, U_{l}, p_{l}\right) & =(1.0,0.0,1.0), \quad x \leq 0.5 \\
\left(\rho_{r}, U_{r}, p_{r}\right) & =(0.125,0.0,0.1), \quad x>0.5
\end{aligned}
$$

The reference mean free path $\ell_{m f p}$ is evaluated by reference state with $p_{\text {ref }}=1.0, \rho_{\text {ref }}=1.0$, and the viscosity coefficient $\mu_{r e f}$, which is defined by

$$
\ell_{m f p}=\frac{16}{5 \sqrt{2 \pi}} \frac{\mu_{r e f}}{\sqrt{\rho_{\text {ref }} p_{\text {ref }}}} .
$$

So, the local mean free path in the gas becomes

$$
\ell_{\text {local }}=\frac{\mu}{\mu_{\text {ref }}} \sqrt{\frac{\rho_{\text {ref }}}{\rho} \frac{p_{r e f}}{p}} \ell_{m f p}
$$

where $\mu=\sqrt{\frac{p}{p_{r e f}} \frac{\rho_{r e f}}{\rho}} \mu_{\text {ref }}$ is determined as hard sphere molecules. For the Sod test case, the initial mean free path of the left state is equal to the reference mean free path, and the mean free path of the right side gas is about 9 times of the left. With the variation of $\mu_{r e f}$, we can basically simulate flows with different degree of rarefaction. In the simulating cases with 
reference viscosity coefficients $\mu_{r e f}=10,1.0,10^{-3}$, and $10^{-5}$, and the corresponding mean free path for the left state of the Sod test become $12.77,1.277,1.277 \times 10^{-3}$, and $1.277 \times 10^{-5}$, which cover the flows from free molecule to continuum one. A useful parameter is the local flow Knudsen number, which is defined by

$$
K n_{\text {local }}=\frac{\ell_{\text {local }}}{\rho} \frac{d \rho}{d x}
$$

For example, for the case of $\mu_{r e f}=1.0$, the local mean free path is much greater than 1 . So, with the unit length of computational domain, the flow is basically free molecule one.

Figure 1 shows the simulation results of density, velocity, temperature, and Knudsen number for the case with $\mu_{r e f}=10$. As shown in the Knudsen number plot, it is basically the free molecule flow. The results from the unified BGK precisely recovers the exact solution of the collision-less Boltzmann equation. Figure 2 shows the same calculation at $\mu_{\text {ref }}=1.0$, where the collision-less, unified BGK, and BGK-DOM results are plotted. From the local Knudsen number plot, it is obvious that most part of the flow is still in the collision-less regime. At $\mu_{\text {ref }}=10^{-3}$, the flow is near continuum flow. Figure 3 shows the solutions from all three schemes, which are unified BGK, BGK-DOM, and BGK-NS. Since the BGK-NS is an accurate Navier-Stokes flow solver [29, 31, 32], its solution can be used as a benchmark NS solution. Figure 3 clearly shows that unified BGK and BGK-DOM can get the NS solution as well. As $\mu_{\text {ref }}$ gets to $10^{-5}$, the flow is basically in the continuum regime. It becomes impossible for BGK-DOM method to get solution due to computational cost. So, figure 4 shows the solutions from unified BGK and BGK-NS only. In this case, unified BGK basically becomes a shock capturing scheme for the Euler solution because the numerical mesh size cannot resolve the physical structure at all.

The second test case is designed as a case which covers flow in the upper and lower transition regimes. The purpose is to show the efficiency of unified BGK and BGK-DOM method. The computational domain is $x \in[0,2]$ with different number of mesh points. The initial condition for this case

$$
\begin{aligned}
\left(\rho_{l}, u_{l}, p_{l}\right) & =(0.001,0.0,0.001), & x \leq 0.8 \\
\left(\rho_{m}, u_{m}, p_{m}\right) & =(10.0,0.0,10.0), & 0.8<x \leq 1.2 \\
\left(\rho_{r}, u_{r}, p_{r}\right) & =(0.001,0.0,0.001), & 1.2<x \leq 2.0
\end{aligned}
$$

We have tested this case with two reference viscosity coefficients $\mu_{\text {ref }}=\left(10^{-3}, 10^{-4}\right)$. The mean free path in the central region and two sides are $\left(1.27 \times 10^{-4}, 1.27 \times 10^{-5}\right)$ and $(1.27,0.127)$. The corresponding collision times are $\left(1.0 \times 10^{-4}, 1\right)$ and $\left(1.0 \times 10^{-5}, 0.1\right)$. 
Therefore, the flows are basically in the upper and lower transition regimes. In this calculation, for the BGK-DOM method, the time step in this calculation is limited to $\Delta t \leq 2 \tau_{m}$, where $\tau_{m}$ is the minimum particle collision time. For the unified scheme, the time step is determined by the CFL condition. As we know, BGK-DOM method is actually an accurate flow solver in all Knudsen number regime. The problem for it is that the time step is limited and the scheme will become extremely expensive in the continuum limit. In the following test, we would like to show that the unified scheme can present accurate solutions with a much larger time step. Also, in order to test the sensitivity of the solution on the numerical mesh size, three meshes with 50,100 and 200 will be used in the domain. The first one is the upper transition flow calculation with $\mu_{r e f}=10^{-3}$. Figure 5 shows the temperature and local Knudsen number distributions by the unified scheme and BGK-DOM in three different mesh sizes. As shown in the figure, with the similar solution the unified scheme can use a larger time step, which is on the order of 10 times. In the lower transition regime with $\mu_{\text {ref }}=10^{-4}$, figure 6 presents temperature and local Knudsen number distributions with three different mesh sizes. As shown, the unified scheme can use a time step which is on the order of 100 times larger than the time step used in BGK-DM method. At the same time, the solutions from unified scheme are more accurate than the BGK-DOM method. For the continuum high Reynolds number continuum flows, the reference viscosity coefficients can be on the order of $10^{-7}, 10^{-8}$, under these conditions, the BGK-DOM method is computationally prohibitive due to its extreme cost. However, under these conditions the time step used by the unified scheme can be $10^{5}, 10^{6}$ times larger than the BGK-DOM time step. Also, in the continuum flow regime, the unified scheme will converge to the BGK-NS method, where the Navier-Stokes solutions can be confidently obtained by the unified method.

\section{Conclusion}

In this paper, we present a unified kinetic approach for flows in the entire Knudsen number. The validity of the approach is based on its full representation of particle movement, i.e., transport and collision. Different from many other approaches, the critical step is that the integral solution of the kinetic model is used in the flux evaluation across the cell interface. The integral solution gives an accurate representation in both continuum and free molecule flows. The current scheme can be considered as a dynamic hybrid method, where the different flow behavior is obtained through the different limits of the integral solution of a single kinetic equation, instead of solving different governing equations in different flow regimes. The weakness for the most current existing kinetic methods is that a purely upwinding technique is used in the flux evaluation for the transport term $u f_{x}$, which is equivalent to solving the collisionless Boltzmann equation and its solution is only a partial solution of the integral solution used in the unified scheme. Theoretically, the Boltzmann equation 
is a statistical model, where the dynamic separation of transport and collision disappears. Both transport and collision processes take place everywhere in space and time. So, there is no reason to believe that these particles which transport across the cell interface will not suffer particle collision during its movement toward the cell interface. Therefore, an "exact" integral solution of the full kinetic equation has to be used and it is the key for the success of the unified scheme.

The numerical tests presented in this paper validate the current approach in both continuum and rarefied flow regime. For the continuum flow at high Reynolds number, a standard CFL condition for the macroscopic NS equations is used to determine the time step for the unified scheme, which is much larger than the particle collision time. Overall, the unified scheme is an AP method for the kinetic BGK equation. The method presented in this paper can be easily extended to 2D and 3D cases. Also, the unified scheme for Shakhov model and full Boltzmann equation will be considered in the near future $[24,6]$.

\section{Acknowledgments}

K. Xu was supported by Hong Kong Research Grant Council 621709, National Natural Science Foundation of China (Project No. 10928205), and US National Science Foundation Grant DMS-0914706. J.C. Huang was supported by National Science Council of Taiwan through grant no. NSC 96-2221-E-019-067-MY2.

\section{References}

[1] V.V. Aristov, Direct Methods for Solving the Boltzmann Equation and Study of Nonequilibrium Flows, Kluwer Academic Publishers (2001).

[2] M. Bennoune, M. Lemou, L. Mieussens, Uniformly Stable Numerical Schemes for the Boltzmann Equation Preserving the Compressible Navier-Stokes Asymptotics, J. Comput. Phys. 227 (2008), pp. 3781-3803.

[3] P.L. Bhatnagar, E.P. Gross, and M. Krook, A Model for Collision Processes in Gases I: Small Amplitude Processes in Charged and Neutral One-Component Systems, Phys. Rev., 94 (1954), pp. 511-525.

[4] G.A. BIRD, Molecular Gas Dynamics and the Direct Simulation of Gas Flows, Oxford Science Publications (1994).

[5] J.F. Bourgat, P. Le Tallec, And M.D. Tidriri, Coupling Boltzmann and NavierStokes Equations by Friction, J. Comput. Phys. 127 (1996), pp. 227-245. 
[6] S. Chapman And T.G. Cowling, The Mathematical Theory of Non-uniform Gases, Cambridge University Press (1990).

[7] C.K. CHu, Kinetic-Theoretic Description of the Formation of a Shock Wave, Phys. Fluids 8, 12 (1965).

[8] F. Coron and B. Perthame, Numerical Passage from Kinetic to Fluid Equations, SIAM J. Numer. Anal. 28, pp. 26-42 (1991).

[9] P. Degond, J.G. Liu, And L. Mieussens, Macroscopic Fluid Models with Localized Kinetic Upscale Effects, Multiscale Model. Simul. 5 (2006), pp. 940-979.

[10] S.M. Deshpande, A Second Order Accurate, Kinetic-Theory Based, Method for Inviscid Compressible Flows, NASA Langley Tech. paper No. 2613 (1986).

[11] F. Filbet And S. Jin, A Class of Asymptotic Preserving Schemes for Kinetic Equations and related problems with stiff sources, preprint (2009).

[12] G. May, B. Srinivasan, and A. Jameson, An Improved Gas-Kinetic BGK FiniteVolume Method for Three-Dimesnional Transonic Flow, J. Comput. Phys. 220 (2007), pp. 856-878.

[13] L. Mieussens, Discrete-Velocity Models and Numerical Schemes for the BoltzmannBGK Equtaion in Plane and Axisymmetric Geometries, J. Comput. Phys. 162 (2000), pp. 429-466.

[14] Q.B. Li And S. Fu, On the Multidimensional Gas-Kinetic BGK Scheme, J. Comput. Phys. 220 (2006), pp. 532-548.

[15] Q.B. Li And K. XU, A High-Order Gas-Kinetic Navier-Stokes Solver, preprint (2009).

[16] Z.H. Li And H.X. Zhang, Gas-Kinetic Numerical Studies of Three-Dimensional Complex Flows on Spacecraft Re-Entry, J. Comput. Phys. 228 (2009), pp. 1116-1138.

[17] V.I. Kolobov, R.R. Arslanbekov, V.V. Aristov, A.A. Frolova, S.A. ZabeLOK, Unified Solver for Rarefied and Continuum Flows with Adaptive Mesh and Algorithm Refinement, J. Comput. Phys. 223 (2007), pp. 589-608.

[18] T. OhwadA, On the Construction of Kinetic Schemes, J. Comput. Phys. 177 (2002), pp. 156-175. 
[19] T. Ohwada And K. Xu, The Kinetic Scheme for Full Burnett Equations, J. Comput. Phys. 201 (2004), pp. 315-332.

[20] T. Ohwada and S. Kobayashi, Management of Discontinuous Reconstruction in Kinetic Schemes, J. Comput. Phys. 197 (2004), pp. 116-138.

[21] S. Pieraccini and G. Puppo, Implicit-Explicit Schemes for BGK Kinetic Equations, J. Scientific Computing 32 (2007), pp. 1-28.

[22] D.I. Pullin, Direct Simulation Methods for Compressible Inviscid Ideal Gas Flow, J. Comput. Phys., 34 (1980), 231-244.

[23] T.E. Schwartzentruber and I.D. Boyd, A Hybrid Particle-Continuum Method Applied to Shock Waves, J. Comput. Phys. 215 (2006), pp. 402-416.

[24] E.M. Shakhov, Generalization of the Krook kinetic Equation, Fluid Dyn. 3, 95 (1968).

[25] M.D. Su, K. Xu, M. Ghidaoui, Low Speed Flow Simulation by the Gas-Kinetic Scheme, J. Comput. Phys., 150 (1999), pp. 17-39.

[26] S. TIWARI, Coupling of the Boltzmann and Euler Equations with Automatic Domain Decomposition, J. Comput. Phys. 144 (1998), pp. 710-726.

[27] E. Toro, Riemann Solvers and Numerical Methods for Fluid Dynamics, Springer (1999).

[28] K. Xu, Numerical Hydrodynamics from Gas-Kinetic Theory, Ph.D. thesis (1993), Columbia University.

[29] K. Xu, A Gas-Kinetic BGK Scheme for the Navier-Stokes Equations and Its Connection with Artificial Dissipation and Godunov Method, J. Comput. Phys. 171 (2001), pp. 289-335.

[30] K. Xu And Z.W. Li, Dissipative Mechanism in Godunov-Type Schemes, Int. J. Numer. Methods in Fluids, vol. 37, pp. 1-22 (2001).

[31] K. Xu, M.L. MaO, and L. Tang, A Multidimensional Gas-Kinetic BGK scheme for hypersonic viscous flow, J. Comput. Phys. 203 (2005), pp. 405-421.

[32] K. Xu, X. He, C. CAI, Multiple Temperature Kinetic Model and Gas-Kinetic Method for Hypersonic Nonequilibrium Flow Computations, J. Comput. Phys. 227 (2008), pp. 6779-6794.

[33] J.Y. Yang and J.C. Huang, Rarefied Flow Computations Using Nonlinear Model Boltzmann Equations, J. Comput. Phys. 120 (1995), pp. 323-339. 

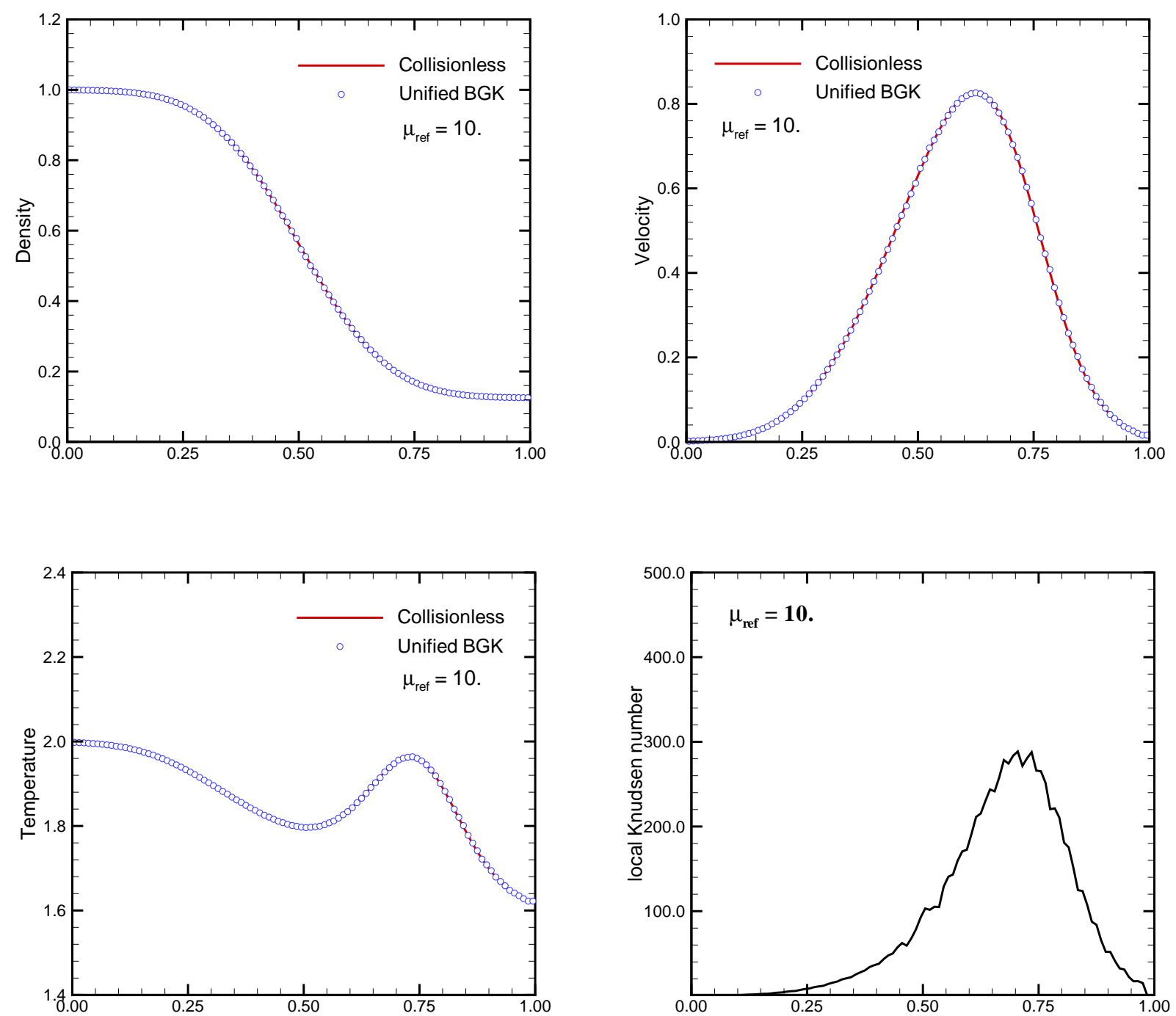

Figure 1: Sod test: Unified BGK and collisionless Boltzmann solutions at $\mu_{r e f}=10$. 

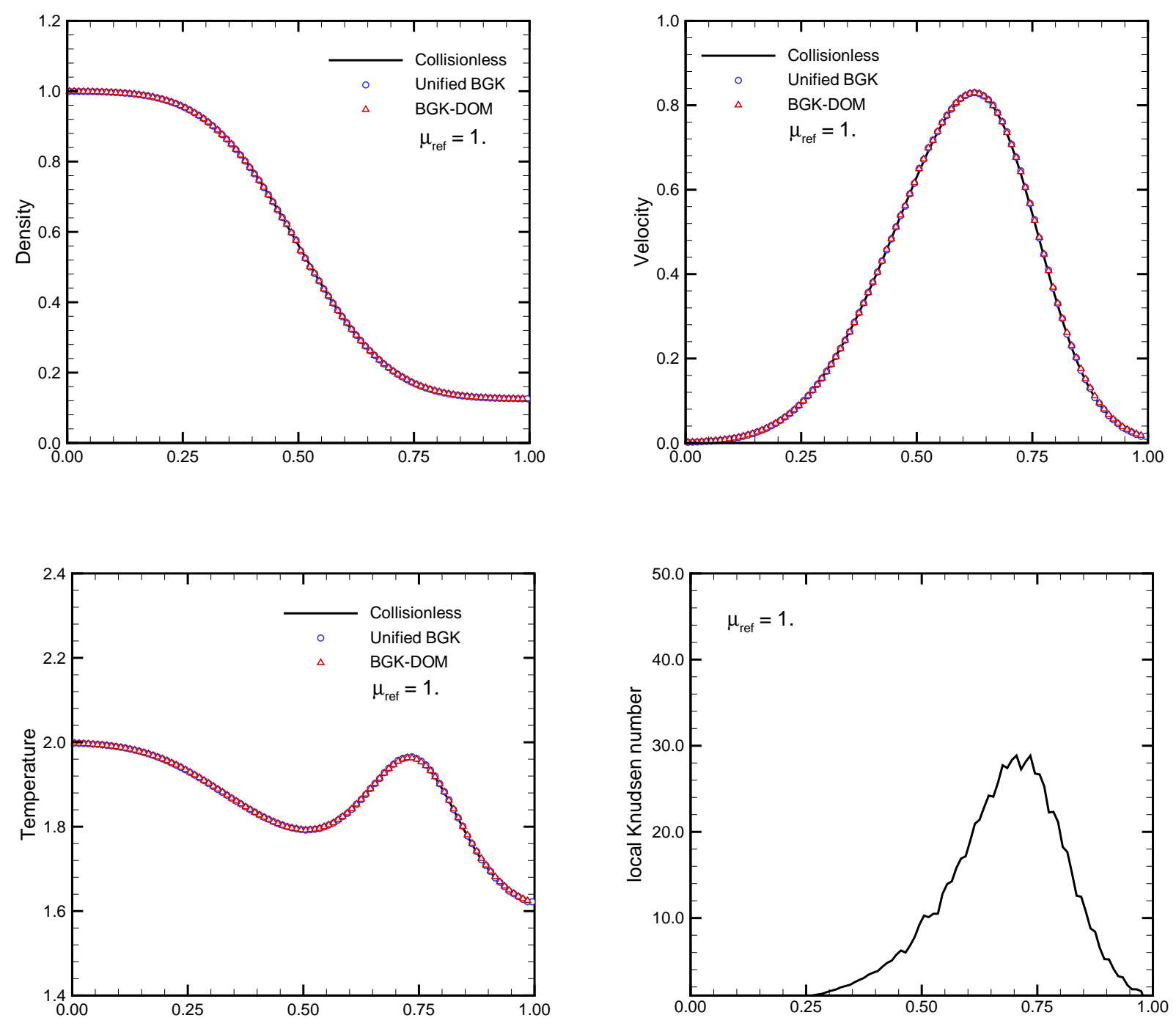

Figure 2: Sod test: Unified BGK, BGK-DOM, and collisionless Boltzmann solutions at $\mu_{r e f}=1$. 

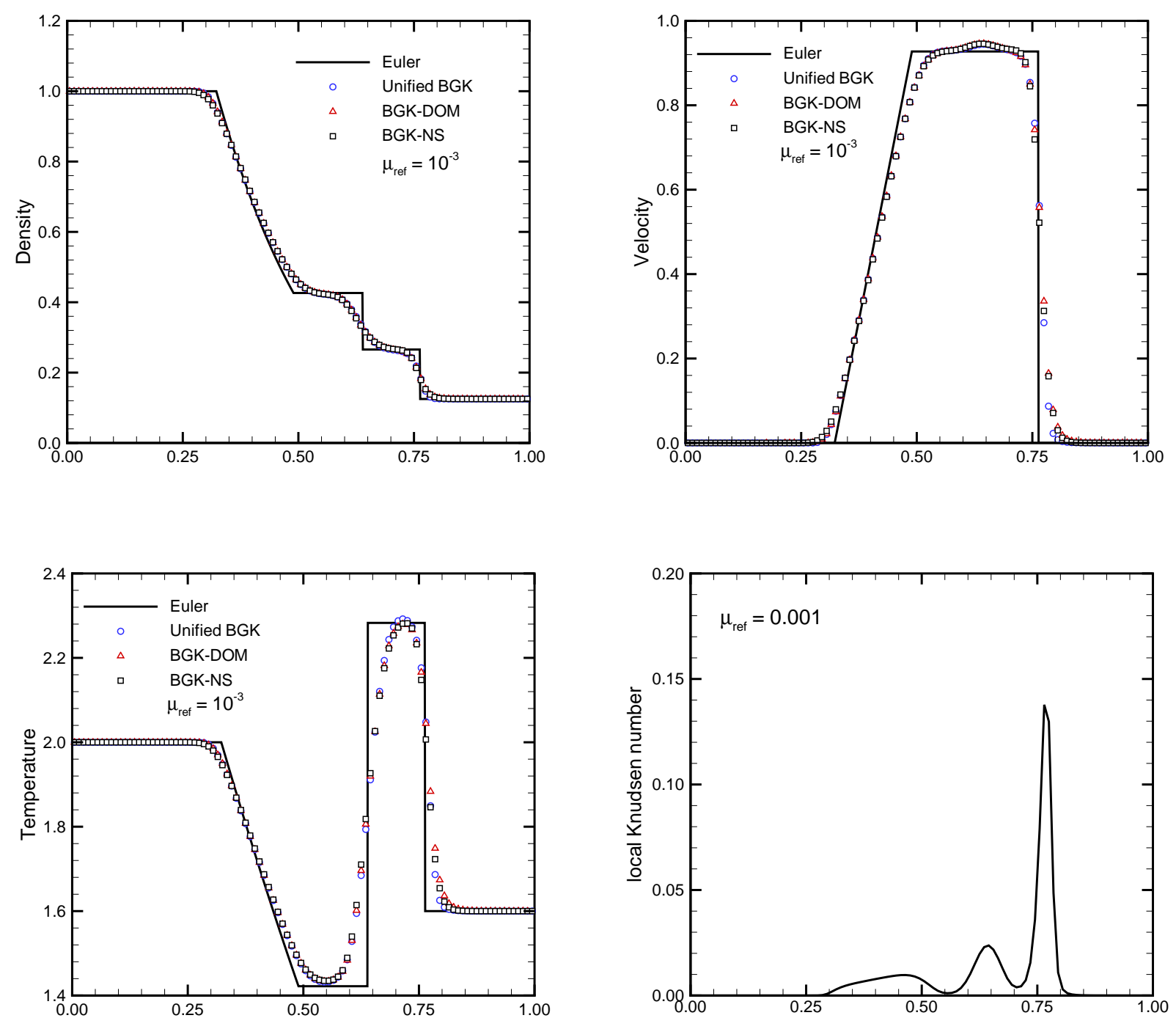

Figure 3: Sod test: Unified BGK, BGK-DOM, BGK-NS, and Euler Solutions at $\mu_{r e f}=10^{-3}$ 

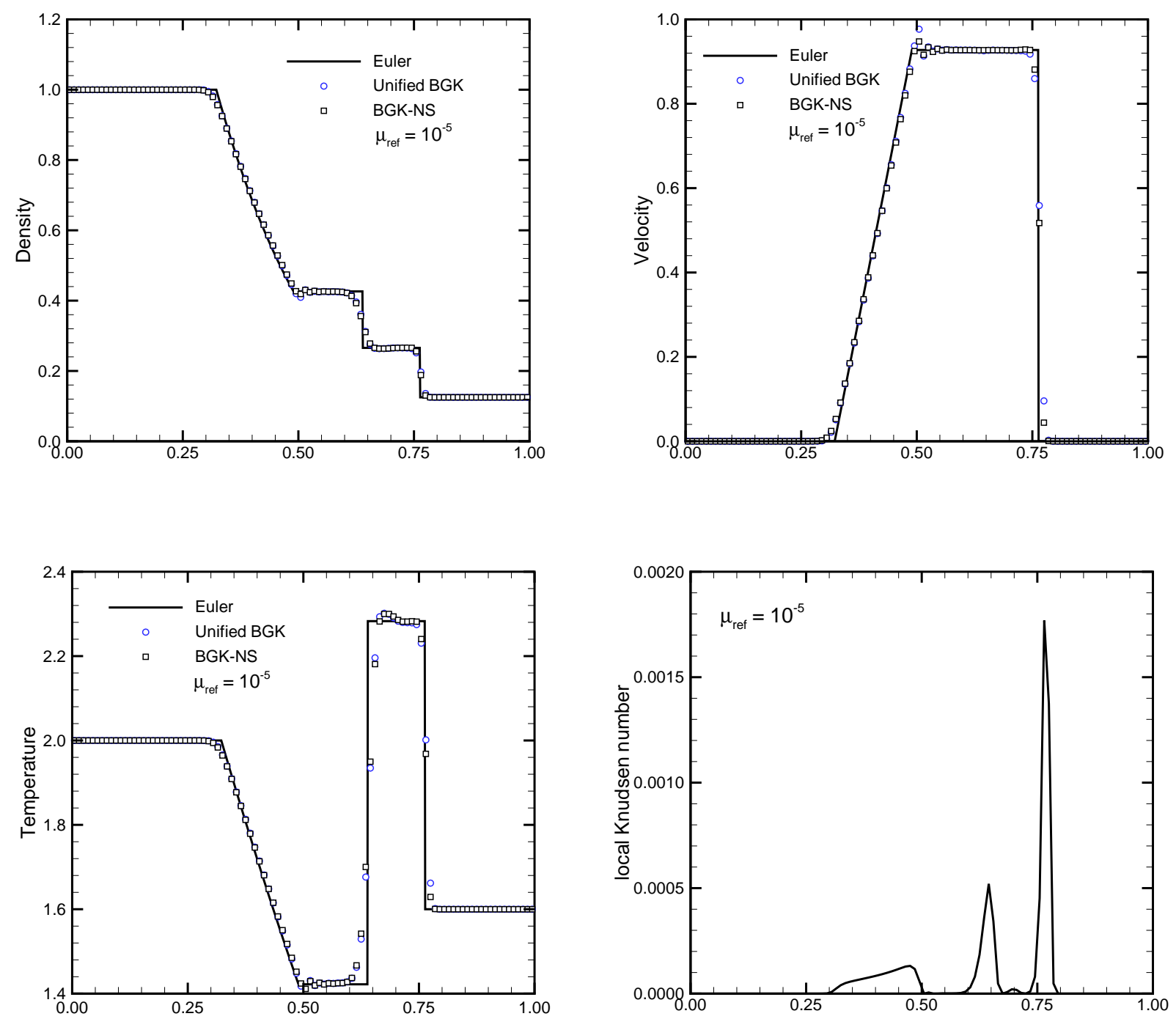

Figure 4: Sod test: Unified BGK, BGK-NS, and Euler solutions at $\mu_{r e f}=10^{-5}$ 

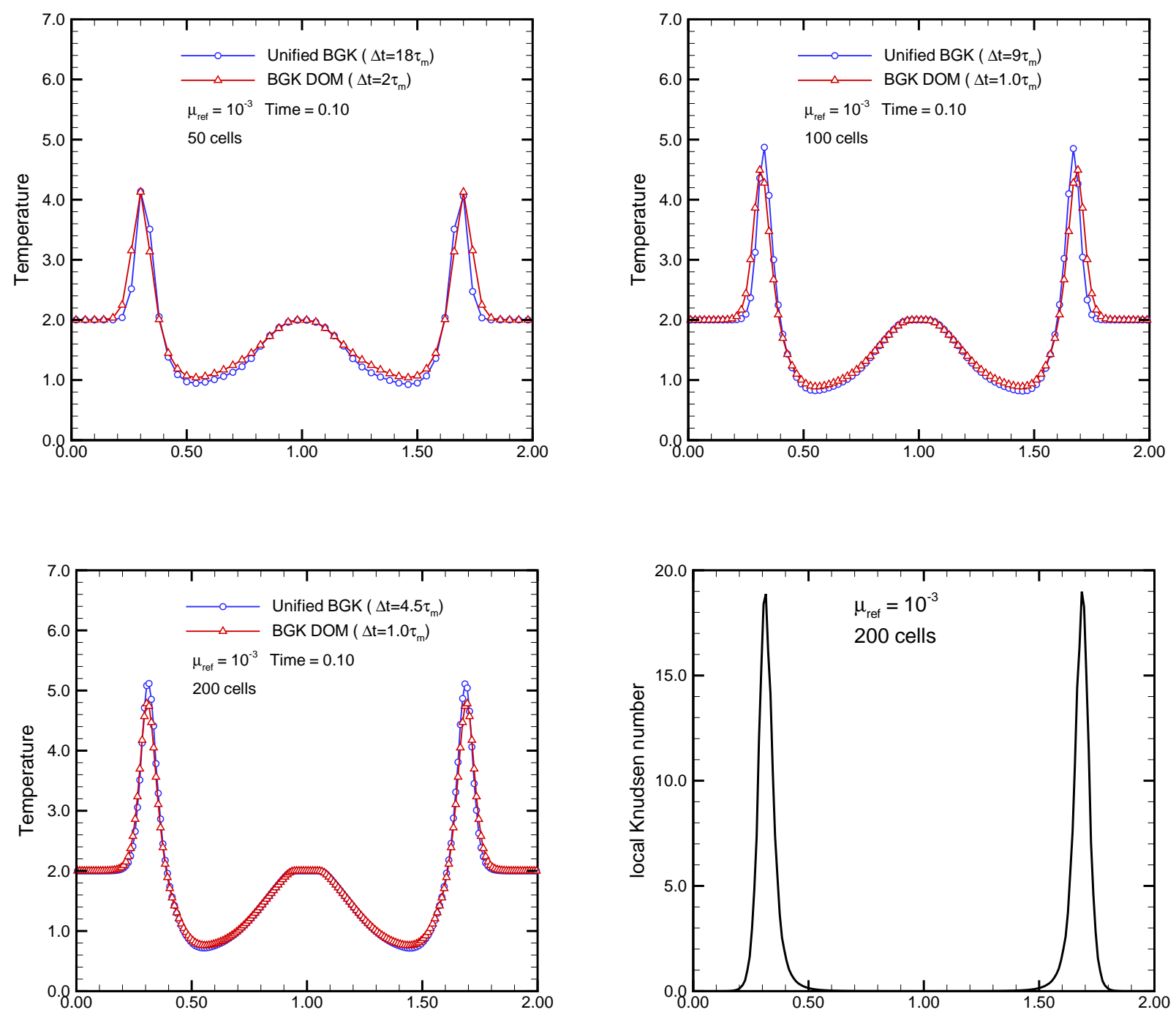

Figure 5: Test: Unified BGK and BGK-DOM solutions with different mesh size and time steps at $\mu_{r e f}=10^{-3}$ 

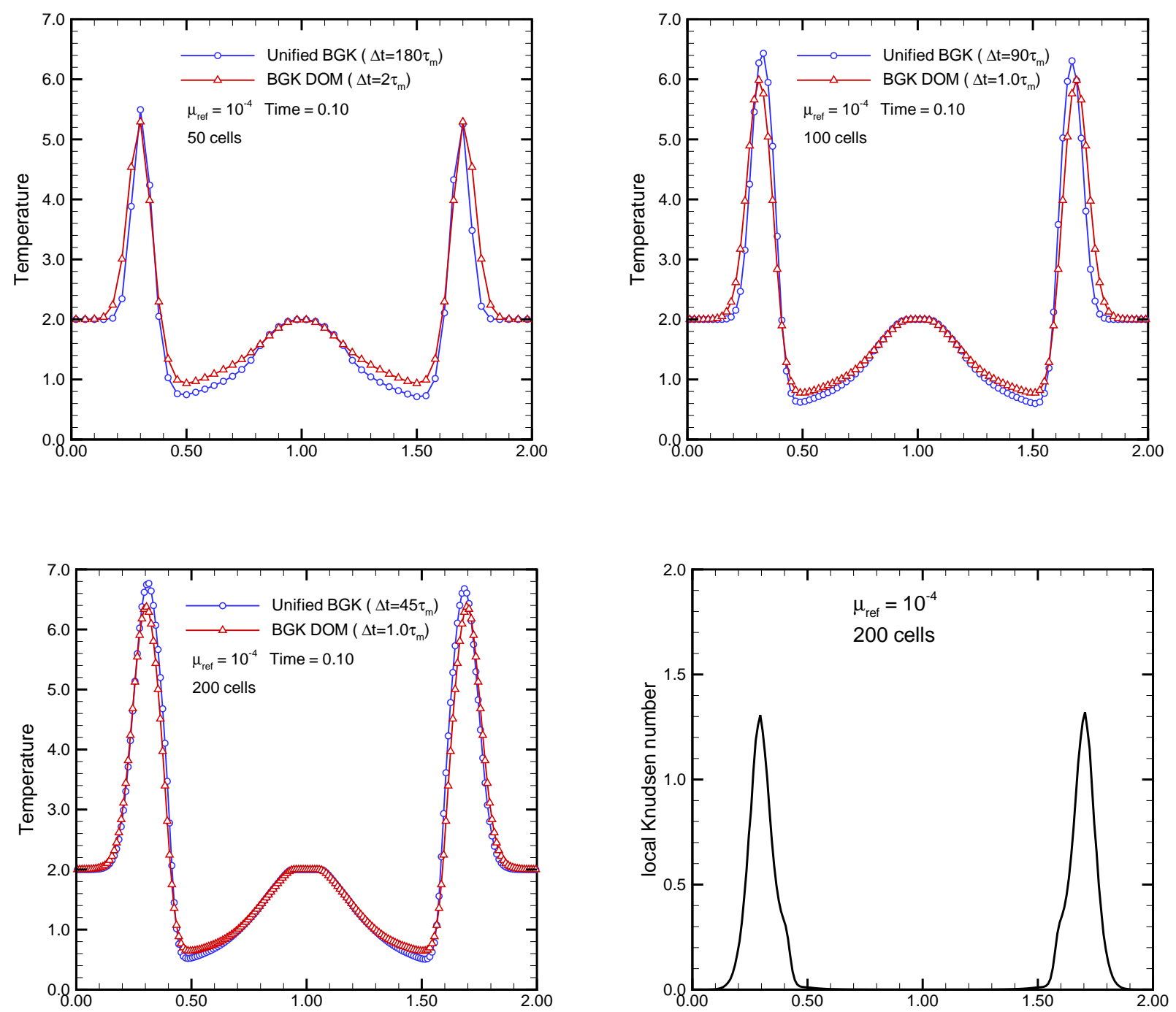

Figure 6: Test: Unified BGK and BGK-DOM solutions with different mesh size and time step at $\mu_{r e f}=10^{-4}$ 